\begin{tabular}{|c|c|c|c|c|c|c|}
\hline Datum & & M.Z.Gr. & $\mathrm{Bb}$. & Gr. & Curve & B. - C. \\
\hline \multirow[t]{6}{*}{97 Nov. } & 12 & $5^{h} 37^{m}$ & M & 6.64 & 6.62 & +2 \\
\hline & I 3 & $5 \quad 55$ & $\mathbf{M}$ & 7.03 & 7.01 & +2 \\
\hline & 13 & $6 \quad 1$ & $\mathrm{~K}$ & 7.04 & 7.01 & +3 \\
\hline & 14 & 719 & $\mathbf{M}$ & $7 \cdot 34$ & 7.27 & +7 \\
\hline & 16 & 535 & M & 6.68 & 6.69 & -1 \\
\hline & 20 & 647 & $\mathrm{~K}$ & 6.74 & 6.77 & -3 \\
\hline \multirow[t]{14}{*}{ Dec. } & 9 & 446 & $\mathbf{M}$ & $6.5^{I}$ & 6.64 & -13 \\
\hline & I 5 & 457 & $\mathrm{~K}$ & $7.4 \mathrm{I}$ & $7 \cdot 30$ & +11 . \\
\hline & 16 & 519 & M & 7.16 & 7.08 & +8 \\
\hline & 17 & $\begin{array}{ll}5 & 23\end{array}$ & $K$ & 6.92 & 6.79 & +13 \\
\hline & 18 & $5 \quad 11$ & M & 7.14 & 7.12 & +2 \\
\hline & 19 & 445 & $\mathrm{~K}$ & 7.23 & $7 \cdot 33$ & -10 \\
\hline & 19 & 447 & $\mathrm{M}$ & 7.30 & $7 \cdot 33$ & -3 \\
\hline & 26 & $44 I$ & $\mathbf{M}$ & 7.22 & $7 \cdot 19$ & +3 \\
\hline & 27 & 441 & $\mathbf{M}$ & $7 \cdot 34$ & $7 \cdot 37$ & -3 \\
\hline & 28 & 446 & $\mathrm{M}$ & 6.70 & 6.62 & +8 \\
\hline & 29 & 40 & $\mathbf{M}$ & $6.9 \mathrm{I}$ & 6.95 & -4 \\
\hline & 29 & 46 & $\mathrm{~K}$ & 6.88 & 6.95 & -7 \\
\hline & $3^{\circ}$ & 414 & M & 7.20 & 7.22 & -2 \\
\hline & $3^{\circ}$ & 420 & $\mathrm{~K}$ & 7.11 & 7.22 & - II \\
\hline \multirow[t]{5}{*}{98 Jan. } & $\mathbf{I}$ & 4 I 8 & $\mathrm{M}$ & 6.53 & $6.6 \mathrm{I}$ & -8 \\
\hline & 4 & 430 & $\mathrm{M}$ & 7.17 & 7.29 & -12 \\
\hline & I 4 & 433 & $\mathrm{~K}$ & 7.24 & 7.14 & +10 \\
\hline & 18 & $44^{6}$ & $\mathrm{M}$ & 7.09 & 7.17 & -8 \\
\hline & I 9 & $5 \quad 17$ & $\mathrm{~K}$ & $7 \cdot 35$ & $7 \cdot 37$ & -2 \\
\hline
\end{tabular}

Die beobachteten Grössen lassen sich hier, soweit man dies bei einer graphischen Ausgleichung beurtheilen kann, durch eine Periode von 3.84 Tagen darstellen; die Hellig. keit im Maximum ist ungefähr $6^{\mathrm{m}} \cdot 6$, im Minimum $7 \cdot 4$. Zieht man wieder auch die beiden weiter zurückliegenden Beobachtungen aus den Jahren 1890 und $189^{6}$ hinzu, von denen die erste sehr nahe einem Minimum, die zweite angenähert einem Maximum entspricht, so findet man als vorläufig plausibelsten Werth für die Periodenlänge 3.844 Tage. Zur Vorausberechnung der Epochen wird bis auf weiteres angenommen werden können:

Maximum $=1897$ Oct. 4.66 mittl. Z. Gr. +3.844 E.
Die Lichtcurve dieses Veränderlichen unterscheidet sich wesentlich von der des ersteren Sterns, da die Zunahme des Lichts viel schneller als die Abnahme vor sich geht, Das Minimum ist von dem vorausgehenden Maximum um etwa 2.9 Tage, dagegen von dem folgenden Maximum nur um etwas über 0.9 Tage entfernt. Die Lichtcurve ist also derjenigen von $\delta$ Cephei sehr ähnlich. Bemerkenswerth ist dabei vielleicht noch, dass ungefäbr $I^{3} / 4$ Tage nach dem Maximum ein Stillstand in der Lichtabnahme angedeutet ist, eine Erscheinung, die auch bei einigen anderen Sternen vom $\delta$ Cephei-Typus bemerkt worden ist. Diese Einbiegung, welche natürlich erst noch weiterer Bestätigung bedarf, ist beim Zeichnen der Normalcurve nicht berücksichtigt worden; sie spricht sich daher auch nicht in der folgenden Tabelle aus, welche die aus der Curve abgelesenen Helligkeiten von $0.2 \mathrm{zu} 0.2$ Tagen enthält.

\begin{tabular}{c|c}
$\begin{array}{c}\text { Entfernung } \\
\text { vom } \\
\text { Maximum }\end{array}$ & Helligkeit \\
\hline odo & $6^{\mathrm{m}} .57$ \\
o.2 & 6.65 \\
0.4 & 6.73 \\
o.6 & 6.82 \\
o.8 & 6.89 \\
I.० & 6.97 \\
I.2 & 7.03 \\
I.4 & 7.08 \\
I.6 & 7.14 \\
I.8 & 7.18
\end{tabular}

\begin{tabular}{c|c}
\hline $\begin{array}{c}\text { Entfernung } \\
\text { vom } \\
\text { Maximum }\end{array}$ & Helligkeit \\
\hline 2.0 & 7.23 \\
2.2 & 7.27 \\
2.4 & 7.31 \\
2.6 & 7.34 \\
2.8 & 7.37 \\
3.0 & 7.37 \\
3.2 & 7.23 \\
3.4 & 7.05 \\
3.6 & 6.85 \\
3.8 & 6.63
\end{tabular}

Aus den Abweichungen der einzelnen Beobachtungen von den nach dieser Tabelle berechneten Helligkeiten ergiebt sich für den wahrscheinlichen Fehler einer Beobachtung der Werth \pm 0 mo6o.

Die Farbe des Sterns liegt nach der in der Potsdamer Durchmusterung eingeführten Farbenscala zwischen GW und WG.

Potsdam, Astrophys. Observatorium, 1897 März 8.

\title{
Die Leoniden und Bieliden von 1897 und 1896.
}

\section{(Mitgetheilt vom Director Prof. F. A.C. Oudemans.)}

Fünf Nächte widmete ich im Jahre 1897 mit der sehr geschätzten Hülfe des Herrn Cand. der Astr. van Lummel, der Beobachtung des Leonidenschwarmes. Am I2., I4. und 16. Nov. war der Himmel fortwährend ganz oder beinahe ganz bewölkt; dagegen waren die Nächte des 13. und I 5. Nov. sehr klar. Bedenkt man die ungünstige Jahreszeit, so kann man meiner Meinung nach mit diesen 2 Nächten zufrieden sein. Leider hinderte der Mond, der erst am I 7. Nov, im letzten Viertel war, besonders dadurch, dass er, wenigstens in der sehr heiteren Nacht rom 15. Nov., zu nahe beim Löwen stand. Es wurden also erstens nur helle Sternschnuppen beobachtet, und überdies wurde die Einzeichnung der Flugbahnen sehr erschwert, weil nur die helleren
Sterne als Anhaltspunkte benutzt werden konnten. Die beobachteten Sternschnuppen wurden auf eine schwarze Kugel (A. N. 3307) gezeichnet, und nachher auf eine specielle, im Jabre 1867 von Professor M. Hoek - in stereographischer Projection gezeichnete, Karte übertragen.

Am 1 3. Nov. beobachtete ich von $12^{\text {h }} 51^{\mathrm{m}}$ bis $16^{\mathrm{h}} 7^{\mathrm{m}}$ I 2 Sternschnuppen (7 Leoniden, 3 Tauriden); am I 5 . Nov. von $13^{\mathrm{h}} 8^{\mathrm{m}}$ bis $16^{\mathrm{h}} 45^{\mathrm{m}} 40$ Sternschnuppen $\left(3^{2}\right.$ Leoniden, 3 Tauriden). Aus den Leoniden stellte sich mit ziemlich grosser Schärfe der Radiant $\alpha=152^{\circ}, \delta=+24^{\circ}$ heraus, während ein secundärer Radiant in $\alpha=150^{\circ}, \delta=+29^{\circ}$ gefunden wurde. 
Von sämmtlichen 39 Leoniden gehörten 8 zur vierten, I I zur dritten, I I zur zweiten und 4 zur ersten Grössenclasse, während $\mathrm{I}$ und ${ }_{4}$ Sternschnuppen die Helligkeit des Jupiter, resp. der Venus hatten. Von den hellsten Leoniden zeigten 4 resp. 5 eine bestimmt grüne, resp. orange Farbe; weitaus die Mehrzahl der Leoniden bewegte sich sehr schnell. Weiter wurde $15 \mathrm{mal}$ im hellen Mondschein eine leuchtende Spur beobachtet, welche bis zu 7 Secunden sichtbar blieb.

Die bedeutende Zahl von hellen und sehr hellen Meteoren lässt vermuthen, dass innerhalb des Utrechter Hori- zontes am 15. Nov. 1897 um etwa $16^{\text {h }}$ ein ziemlich reicher Schwarm von Leoniden sichtbar war, der vielleicht der zweite (folgende) Nebenschwarm (Observatory 1897, S. 340) gewesen sein mag.

Es sei mir gestattet, hier kurz das Wenige zu erwähnen, das ich in Jahre 1897 von Bieliden und im Jahre 1896 von Bieliden und Leoniden habe beobachten können. Die heiteren Abende waren äusserst selten; die sehr dürftigen Ergebnisse sind aus der hier folgenden Tabelle ersichtlich.

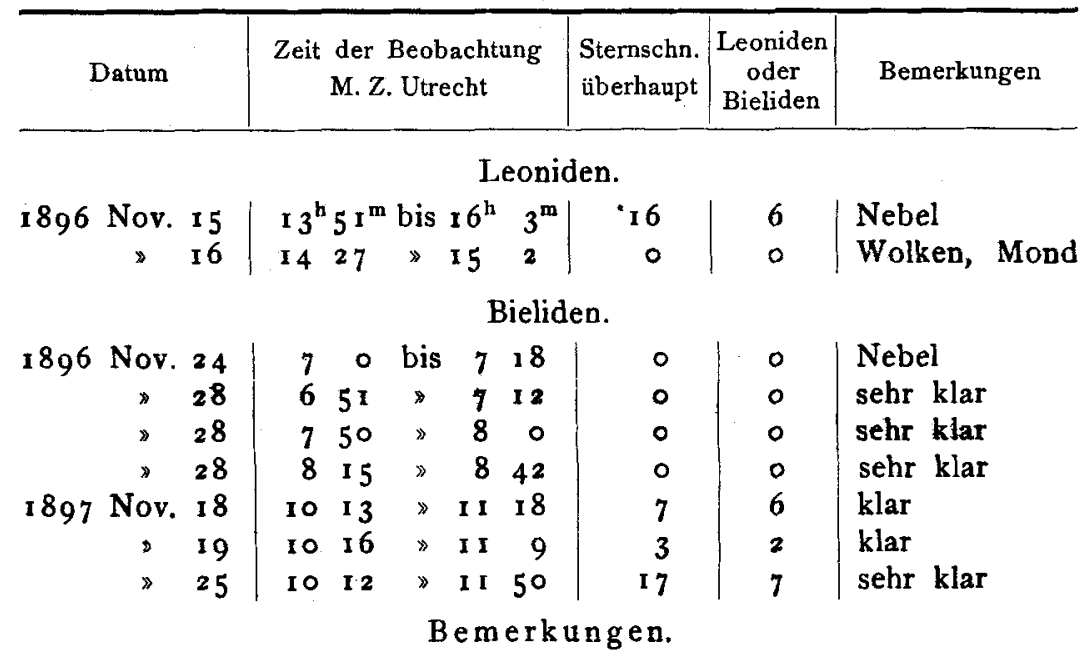

1896 Nov. 15 . Von den 6 beobachteten Leoniden zeigten 5 eine leuchtende Spur; mit Ausnahme von I bewegten sie sich alle schnell.

I 896 Nov. 28 . Um $8^{\text {h }} 53^{\mathrm{m}}$ beim Nachbausegehen sah ich zwischen Wolken eine glänzende Bielide $(=4)$ im Pegasus.

Utrecht, $\times 898$ Jan. 4 .

Dr. A. A. Nijland.

\section{Bestimmungen von Fixsternparallaxen.}

In den Jahren 1887 bis 1892 habe ich am Heliometer der Leipziger Sternwarte neben anderen Beobachtungen auch von einer Anzahl von Sternen mit starker Eigenbewegung die Parallaxe bestimmt. Diese Arbeiten sind in Bd. XXII (Nr. IV) und Bd. XXIV (Nr. III) der Abhandlungen der

math.-phys. Classe der K. S. Gesellschaft der Wissenschaften veröffentlicht worden. Indem ich betreffs Einzelheiten auf diese Abhandlungen verweise, theile ich nachstehend kurz die erlangten Resultate mit.

\begin{tabular}{ll|c|cc|c|c|c}
\hline \multicolumn{1}{c|}{ Stern } & & E.B. & $\pi$ & $\varepsilon$ & $Z$ & $\varepsilon^{\prime}$ & Vergleichsterne \\
\hline y Cassiopejae & $4^{\mathrm{m}}$ & 1.20 & +0.18 & 0.030 & 45 & 0.15 & $+56^{\circ 1112+}+57^{\circ} 172$ \\
$\mu$ Cassiopejae & 5.5 & 3.74 & +0.13 & 0.037 & 23 & 0.16 & $+53.207+54.241$ \\
Lal. 15290 & 8.5 & 1.97 & +0.02 & 0.043 & 32 & 0.16 & $+31.1648+30.1620$ \\
Lal. 18115 praec. & 8.0 & $\cdots$ & +0.18 & 0.027 & 22 & 0.11 & \\
Lal. 18115 seq. & 8.0 & $\ldots$ & +0.18 & 0.032 & 21 & 0.12 & $+53.1309+53.1330$ \\
Lal. 18115 med. &. & 1.69 & +0.18 & 0.020 & 43 & 0.11 & \\
$\vartheta$ Ursae maj. & 3 & 1.11 & +0.09 & 0.035 & 22 & 0.14 & $+52.1389+51.1536$ \\
AOe. 10603 & 6.5 & 1.45 & +0.17 & 0.013 & 27 & 0.12 & $+50.1707+49.1946$ \\
$\beta$ Comae & 4 & 1.20 & +0.11 & 0.042 & 42 & 0.18 & $+28.2207+28.2184$ \\
31 Aquilae & 5.5 & 0.96 & +0.06 & 0.015 & 40 & 0.16 & $+11.3802+12.3929$ \\
Bradley 3077 & 6 & 2.08 & +0.13 & 0.012 & 39 & 0.14 & $+56.2956+56.2978$
\end{tabular}

Die Eigenbewegungen im Bogen grössten Kreises (E. B.) sind nach dem Berliner Jahrbuche resp. nach Porter berechnet. Neben den Parallaxen sind unter $\varepsilon$ die mittleren Fehler derselben vermerkt. $Z$ giebt die Anzahl der Beobachtungsabende an, auf denen das Resultat beruht. $\varepsilon^{\prime}$ ist der mittlere Fehler eines Abendresultates. In der letzten Spalte sind die beiden Vergleichsterne nach der BD. angeführt, gegen welche die relative Parallaxe des betreffenden Sterns bestimmt worden ist. 